Voix et Images

\title{
Je suis écrivain avant tout. Entretien avec Daniel Gagnon
}

\section{Agnès Whitfield}

Volume 18, numéro 1 (52), automne 1992

Les écritures masculines

URI : https://id.erudit.org/iderudit/200998ar

DOI : https://doi.org/10.7202/200998ar

Aller au sommaire du numéro

Éditeur(s)

Université du Québec à Montréal

ISSN

0318-9201 (imprimé)

1705-933X (numérique)

Découvrir la revue

Citer ce document

Whitfield, A. (1992). Je suis écrivain avant tout. Entretien avec Daniel Gagnon.

Voix et Images, 18(1), 62-66. https://doi.org/10.7202/200998ar d'utilisation que vous pouvez consulter en ligne.

https://apropos.erudit.org/fr/usagers/politique-dutilisation/ 


\title{
Je suis écrivain avant tout. Entretien avec Daniel Gagnon
}

\author{
Agnès Whitfield, Université York
}

\section{V. et I. - L'écriture a-t-elle un sexe?}

D. G. - L'écriture n'a pas vraiment de sexe. Il est remarquable que les procédés de différenciation qu'emploie l'écriture d'un sexe ou l'autre pour nous communiquer sa vision d'un monde séparé entre homme et femme soient à ce point factices et inopérants. Car, selon ces théories, ce n'est pas tant d'écrire qu'il s'agirait, mais de prouver qu'on écrit comme homme ou comme femme, comme si l'écriture n'était rien en elle-même et par elle-même, et ne prenait quelque réalité qu'à se sexuer. N'est-ce pas mettre la charrue avant les bœeufs? N'y a-t-il pas d'abord l'écriture, et ensuite le sexe, plutôt que le sexe et ensuite l'écriture?

S'il en est ainsi, je veux dire si l'écriture existe d'abord et qu'ensuite vienne le sexe qui la colore, eh bien! il n'y a pas lieu de parler vraiment d'écriture masculine, n'est-ce pas? S'il y avait une écriture masculine, ce serait seulement celle qui oublie complètement qu'elle est masculine, qui n'y pense pas, qui ne s'applaudit pas narcissiquement, qui surtout n'en fait pas la promotion et qui ne s'en félicite pas. L'écriture existè avant d'être particularisée, individualisée.

Par exemple, l'auteur se tient à la hauteur universelle de son écriture avant d'appartenir à sa nation. L'écriture doit exister d'abord, avant l'engagement nationaliste, ce n'est que par la suite que la nationalité teintera forcément ses textes. Mieux encore, l'œuvre sera d'autant plus nationale qu'elle sera bien écrite. Un écrivain qui écrit bien, je veux dire un écrivain qui est écrivain, est plus important pour son pays qu'un écrivain engagé, nationaliste, féministe ou marxiste ou tout ce que l'on voudra, car ce dernier risque tout simplement d'être un propagandiste, un politicien, un tribun plutôt qu'un écrivain. La parole existe d'abord, ensuite elle passe par le filtre particulier de la culture, hongroise, espagnole ou japonaise, elle se traduit, cette parole originelle, dans l'être national. Ainsi en est-il, à mon avis, de son passage 
dans l'être sexué. C'est dans ce sens seulement que l'on pourrait parler d'écriture masculine et d'écriture féminine.

v. et I. - Alors, il existe quand même des écritures masculines, des écritures féminines?

D. G. - Il n'y pas l'écriture féminine d'un côté et l'écriture masculine de l'autre. Il faudrait plutôt parler d'un auteur masculin, ou d'un auteur féminin se servant de l'écriture, comme un homme ou une femme peuvent se tenir devant l'océan tous deux avec les mêmes droits, comme un homme et une femme peuvent vivre sous le même ciel et s'en inspirer également, comme un homme et une femme peuvent parler d'amour. S'il y a plusieurs amoureux, il n'y a que l'amour, s'il y a plusieurs auteurs, il n'y a que l'écriture.

V. et I. - Mais que dire de toutes ces injustices qui ont été faites à la parole féminine?

D. G. - L'écriture n'a souffert de rien. Les femmes. ont souffert, et les hommes aussi, mais l'écriture n'a rien perdu, rien gagné. De cette confusion vient sans doute le malaise que les féministes créent chez nous par ricochet, fort semblable à celui qui nous prend à la lecture d'œuvres de propagande, et que nous n'éprouvons aucunement devant les œuvres écrites purement et simplement par des femmes; comme si le désir profond de valoriser à tout prix l'écriture féminine, qui n'existe pas, désir de démonstration occultant toute liberté d'écrire et étouffant l'imagination parce qu'il vient avant le désir d'écrire et le désir d'imaginer, comme si ce désir pouvait tromper le lecteur ou la lectrice et faire croire qu'une œuvre peut être faite d'arguments. L'œuvre existe avant la preuve. La preuve, s'il y en a jamais une, découle de l'œuvre, et non l'œuvre de la preuve. On ne fera jamais un roman d'une thèse. L'œuvre peut être un cri à l'injustice faite aux femmes par exemple, comme celle faite au Tibétains, et là nous croyons, nous aimons, nous vivons dans l'œuvre. Mais nous aimons les Tibétains, ce sont eux que nous aimons, pas leur bouddhisme, ce n'est pas leur religion, ni leur propagande qui nous fera pleurer ou rire avec eux. Devant ces personnages nous nous sentons touchés. La raison de l'emprise qu'exerce une œuvre sur le lecteur et la lectrice vient de ce qu'elle propose l'image vivante d'un personnage, masculin ou féminin, qu'importe, que nous portons en nous, qu'elle nous transforme en complice fasciné par l'être humain, qu'il soit homme ou femme, à la fin, on dirait qu'on ne fait plus de distinction quand on aime profondément. Par exemple, je voulais une fille comme troisième enfant, et quand mon fils naquit, mon désir s'effaça comme par enchantement, je ne pensai plus à ce désir qui me parut tout à coup artificiel, 
car la vie prenait le dessus, la vie était là d'abord, le reste était secondaire. D'ailleurs, dans l'évolution, les différences sexuelles tendent à s'amenuiser, un jour nous n'aurons plus besoin de sexe, il n'y aura sans doute qu'un seul être asexué, c'est-à-dire portant les deux sexes partout en lui, en elle.

V et I. - Ainsi, selon vous, il n'y pas lieu de cbercher des différences sexuelles dans l'écriture?

D. G. - L'écriture en effet n'est pas différente à la source, elle le devient parce que nous vivons encore dans un monde où les sexes sont séparés. Si l'écriture n'est ni masculine, ni féminine, ou si elle est les deux, à l'origine, il n'en va pas de même pour les auteurs, nous savons très bien qu'il y a des auteurs féminins et des auteurs masculins. C'est là à mon avis que doit se situer le sujet de notre recherche. À la question: "Y a-t-il des auteurs masculins et y a-t-il des auteurs féminins?: on peut répondre oui. Ce ne fut pas toujours le cas. George Sand, ce * cher grand homme - comme l'appelait affectueusement Gustave Flaubert, a répondu *oui, il y a des auteurs féminins, je prends le nom d'un homme pour le dire. Mais l'écriture de Sand n'est pas plus féminine que masculine, son écriture est écriture et c'est ainsi que nous la lisons, c'est parce qu'elle écrit qu'elle est un auteur... et il se trouve que c'est une femme qui écrit, cela n'est pas plus important que cela, à preuve elle n'a jamais été malheureuse de s'appeler George, elle ne voulait pas faire la promotion de quoi que ce soit, non, ce qu'elle voulait c'était surtout écrire:

\section{V. et I. - Ainsi l'écriture masculine n'existe pas?}

D. G. - Non, l'écriture masculine n'existe pas, non plus que l'écriture féminine. La seule façon de comprendre que cette question de l'écriture masculine ait pu être prise au sérieux c'est de s'apercevoir du soupçon de connivence automatique avec le sexe, le vrai, que l'on a fait peser sur l'écriture des auteurs masculins ou féminins, entendant par là la volontaire ou l'involontaire complaisance que l'écrivain ou l'écrivaine apporterait à parler de son sexe, c'est-à-dire de son organe même. Car lors même que l'auteur voudrait ne pas se distinguer, il est bien difficile de cacher un sexe, n'est-ce pas? Si pourtant l'auteur masculin trempait sa plume dans son sperme, aurions-nous là une œuvre plus masculine? Si l'auteur féminin mouillait ses pages de toutes ses humeurs, lirions-nous là une œuvre plus féminine? Qu'estce qui nous empêche, nous les auteurs, de changer de peau, de parler des parties des hommes ou des femmes, le plaisir sexuel n'est-il pas le même? Qu'est-ce que ça change? Quel auteur n'est pas capable de composer une lettre d'une femme à son amant en même temps que la 
réponse de celui-ci, qui n'a pas réussi la transposition sexuelle bien des fois dans l'histoire littéraire? Nous n'avons pas à apprécier ici la masculinité ou la féminité d'un texte, mais la limpidité de son art, sa beauté.

V. et I. - Mais n'aime-t-on pas toujours savoir si l'cuvre est d'une femme ou d'un bomme? Ou est-ce une simple curiosité?

D. G. - Simple curiosité qui n'a rien à voir avec l'œuvre sans doute. Cela sera important quand nous voudrons, après avoir lu le poème ou le roman, connaître la vie de l'auteur. Son sexe et sa date de naissance seront des informations tout autant intéressantes. Si l'auteur est né en juillet, par exemple, nous demanderons-nous s'il a une écriture de Lion, une écriture de Vierge ou de Sagittaire? Sans doute y a-t-il des auteurs Lion, Vierge et Sagittaire, mais il n'y a pas d'écriture Lion, Vierge ou Sagittaire.

V. et I. - Croyez-vous que vos nouvelles et vos romans auraient pu être écrits par une femme?

D. G. - Je crois que oui. Pourquoi n'auraient-ils pas pu l'être? Jean-leMaigre de Marie-Claire Blais et Bérénice Einberg de Réjean Ducharme sont très crédibles, très vraisemblables, ces personnages ont autant de force l'un que l'autre. Un auteur féminin a mis au monde un petit personnage masculin d'une grande vigueur, un auteur masculin a fait naître une petite fille d'une belle force. On m'a souvent reproché d'inventer des personnages féminins, comme si je:les volais aux auteurs féminins, comme si les personnages féminins étaient la chasse gardée des auteurs féminins. Pourquoi écrire seulement des rôles féminins si on est une femme et seulement des rôles masculins si on est un homme? On n'a jamais vu cela au thêâtre. Nous serions conduits à une extrême complaisance envers notre sexe s'il fallait ne parler que de la moitié de soi-même, si nous recherchions avant tout en nos personnages notre propre sexe. Il faut distinguer ici du pur narcissisme le talent créateur, qu'il soit féminin ou masculin. Ce n'est pas parce qu'on est une femme qu'on peut faire vivre une femme dans un roman, c'est tout simplement parce qu'on sait écrire, peu importe le reste.

V. et $\mathbf{L}$ - La relation que vous avez eue avec votre mère n'aurait pas été la même si vous aviez été une fille. Ainsi peut-on dire que votre écriture porte la marque de cette affection privilégiée de la mère pour son fils ou à l'inverse peut être de son absence douloureuse?

D. G. - La même relation est vécue entre le père et la fille, donc tout est pareil, non? Il suffit seulement de traduire d'un sexe à l'autre. 
Peindre les sentiments féminins ou masculins est faire ouvre d'art, il importe seulement que ces sentiments donnent lieu à une sorte de ballet enchanteur, à une danse des masques. J'ai souvent inversé les rôles féminins et masculins dans mes nouvelles. Les rôles sont interchangeables, parfois c'est le petit garçon qui abhorre son père et adore sa mère, parfois c'est la petite fille qui déteste sa mère et idolâtre son père. Cela n'a pas d'importance, la psychologie ne m'intéresse que parce qu'elle me permet une création.

V. et $\mathbf{L}-$ C'est la création qui importe avant tout?

D. G. - Je suis un écrivain avant tout. À quel moment est-ce que je deviens un homme ou une femme, je ne sais trop. J'ai déjà écrit des poèmes d'amour à une femme, mais ces poèmes avaient une voix féminine et s'adressaient à un garçon. Ma correspondante savait lire ces poèmes comme s'ils avaient été écrits pour son propre sexe.

V. et I. - Les femmes ont longtemps été absentes de la littérature, vous croyez que l'écriture en a souffert?

D. G. - L'écriture est du domaine public, personne, homme ou femme, ne peut se l'accaparer. L'écriture n'a pas souffert de l'absence des femmes dans les siècles passés et ne souffrirait pas de l'absence des hommes dans les 'siècles à venir. L'écriture existe par elle-même, que la plume soit tenue par une femme ou par un homme. L'écriture est. Dans l'écriture, je ne suis ni homme ni femme, je suis un être d'avant le sexe. 